\title{
Visual contrast response functions in Parkinson's disease: evidence from electroretinograms, visually evoked potentials and psychophysics
}

\author{
T. Langheinrich ${ }^{\mathrm{a}}$, L. Tebartz van Elst ${ }^{\mathrm{b}}$, W.A. Lagrèze ${ }^{\mathrm{c}}$, M. Bach ${ }^{\mathrm{c}}$, C.H. Lücking ${ }^{\mathrm{a}}$, \\ M.W. Greenlee ${ }^{a, *}$ \\ ${ }^{a}$ Neurologische Universitätsklinik, Universität Freiburg, Breisacherstrasse 64, 79106 Freiburg, Germany \\ ${ }^{\mathrm{b}}$ Psychiatrische Universitätsklinik, Universität Freiburg, Freiburg, Germany

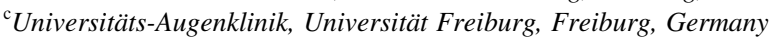

Accepted 16 August 1999

\begin{abstract}
Objectives: Visual contrast detection thresholds and suprathreshold contrast discrimination thresholds were compared to luminance and flash/pattern electroretinograms (ERG) and visually evoked potentials (VEP) in patients with Parkinson's disease ( $n=31$ ), patients with multiple system atrophy $(n=6)$, patients with progressive supranuclear palsy $(n=6)$ and control patients without central nervous disease $(n=33)$.

Methods: The stimuli were luminance modulated full-field (flash) or horizontally oriented sinewave gratings (pattern), the latter having either a low $(0.5 \mathrm{cycles} / \mathrm{deg})$ or medium $(4.0 \mathrm{cycles} / \mathrm{deg})$ spatial frequency. Stimulus contrast ranged from 10 to $80 \%$ so that contrast response functions could be derived.

Results: Contrast thresholds were higher in the patients with Parkinson's disease than in the control patients. Contrast discrimination thresholds were also somewhat elevated in patients with Parkinson's disease. Pattern ERG amplitudes were significantly reduced in patients with Parkinson's disease for the medium spatial frequency stimulus, but less for the low spatial frequency and flash stimuli.

Conclusions: Our results suggest that Parkinson's disease impairs contrast processing in the retina. VEP amplitudes did not significantly differ between the groups for the conditions tested. Patients with progressive supranuclear palsy also showed impaired contrast perception and reduced ERG amplitudes, whereas patients with multiple system atrophy were less impaired. (C) 2000 Elsevier Science Ireland Ltd. All rights reserved.
\end{abstract}

Keywords: Contrast discrimination; Electroretinogram; Visual evoked potentials; Parkinson's disease; Neurodegenerative diseases

\section{Introduction}

There is substantial evidence that Parkinson's disease is associated with visual impairments. These impairments are usually thought to be a result of an attenuation of the retinal signal at an early stage in the visual process. Electrophysiological evidence of visual pathology has been related to delays in visually evoked potentials (Bodis-Wollner and Yahr, 1978; Delwaide, 1980; Gawel, 1981; Marx et al., 1986), as well as amplitude reductions in the pattern-electroretinogram (Ghilardi et al., 1989; Ikeda et al., 1994). Psychophysically-defined contrast detection thresholds are also elevated in patients with Parkinson's disease (BodisWollner et al., 1987; Bodis-Wollner, 1990; Mestre et al., 1990, 1992; Masson et al., 1993). The selective nature of the

\footnotetext{
* Corresponding author. Tel.: +49-761-270-5312; fax: +49-761-270-
} 5310 . visual impairments (medium-to-high spatial frequencies and medium temporal frequencies; (Marx et al., 1986; Bodis-Wollner et al., 1987)) suggests the involvement of specific mechanisms underlying the transfer of pattern contrast. The presence of normal pattern adaptation effects (Tebartz Van Elst et al., 1997) suggests that function in the visual cortex remains intact.

Dopamine is an important neurotransmitter in the visual pathway (Bodis-Wollner et al., 1987). The presence of dopamine in the mammalian retina has been well documented (Haggendal and Malmfors, 1963; Dowling and Ehinger, 1975; Mariani et al., 1984; Massey and Redburn, 1987; Nguyen-Legros et al., 1988; Skrandies and Wässle, 1988), including the human retina (Frederick et al., 1982). In addition to the loss of dopaminergic neurons in the pars compacta of the substantia nigra, Parkinson's disease has also been associated with a reduction in the level of dopamine in the retina (Harnois and Di Paolo, 1990). There is 
some evidence that dopamine might also act at synapses in the lateral geniculate nucleus (Papadopoulos and Parnavelas, 1990) and the visual cortex (Reader and Quesney, 1986; Parkinson, 1989). Thus, dopaminergic activity in the visual system could be diminished in Parkinson's disease.

In an attempt to identify the neural mechanisms underlying the impairment in contrast vision in Parkinson's disease, we recorded electroretinograms (ERGs) and visually evoked potentials (VEPs) in 31 patients with Parkinson's disease. Parallel determination of the contrast detection and discrimination thresholds for two spatial frequencies and five contrast levels was also conducted. We could therefore evaluate the extent to which changes in electrophysiological measurements are associated with concomitant changes in visual performance. The results from patients with Parkinson's disease are compared to those from 33 control patients and 12 patients with related neurodegenerative diseases ( 6 patients with multiple system atrophy, 6 patients with supranuclear palsy) to determine the extent to which the measured changes are specific to this neurodegenerative disorder.

\section{Materials and methods}

\subsection{Subjects}

Table 1 gives a description of the 3 patient groups. All

Table 1

Clinical data on the patients with Parkinson's disease (PD), multiple system atrophy (MSA) and progressive supranuclear palsy (PSP)

\begin{tabular}{|c|c|c|c|c|c|c|}
\hline Patient & Gender & Age & Webster & $\begin{array}{l}\text { Medication } \\
l \text {-Dopa (mg) }\end{array}$ & $\begin{array}{l}\text { Acuity right } \\
\text { eye/left eye }\end{array}$ & Other \\
\hline PD01 & $\mathrm{F}$ & 64 & 4 & $<500$ & $0.6 / 0.8$ & - \\
\hline PD02 & M & 47 & 2 & $<500$ & $1.0 / 1.0$ & - \\
\hline PD03 & $\mathrm{F}$ & 70 & 11 & $<750$ & $0.5 / 0.5$ & - \\
\hline PD04 & $\mathrm{F}$ & 48 & 4 & $<375$ & $0.9 / 1.0$ & - \\
\hline PD05 & M & 66 & 8 & $>750$ & $0.8 / 0.8$ & - \\
\hline PD06 & M & 69 & 15 & $<375$ & $0.6 / 0.0$ & Amaurosis left \\
\hline PD07 & M & 63 & 4 & $<750$ & $1.0 / 0.9$ & - \\
\hline PD08 & M & 57 & 6 & $>750$ & $0.8 / 0.8$ & - \\
\hline PD09 & $\mathrm{F}$ & 54 & 5 & $<750$ & $1.2 / 1.2$ & - \\
\hline PD10 & M & 56 & 10 & Untreated & $0.7 / 0.4$ & - \\
\hline PD11 & M & 71 & 17 & $>750$ & $0.9 / 0.9$ & - \\
\hline PD12 & $\mathrm{F}$ & 63 & 24 & $<750$ & $0.6 / 0.9$ & - \\
\hline PD13 & M & 65 & 11 & $>750$ & $0.6 / 0.8$ & - \\
\hline PD14 & $\mathrm{F}$ & 68 & 9 & $<750$ & $1.0 / 1.0$ & - \\
\hline PD15 & M & 62 & 11 & $>750$ & $1.0 / 1.0$ & - \\
\hline PD16 & M & 37 & 2 & Untreated & $1.2 / 1.1$ & - \\
\hline PD17 & M & 55 & 14 & $<750$ & $0.8 / 0.8$ & - \\
\hline PD18 & M & 64 & 8 & $<500$ & $1.0 / 1.0$ & - \\
\hline PD19 & $\mathrm{F}$ & 44 & 9 & $<500$ & $1.0 / 1.0$ & - \\
\hline PD20 & M & 46 & 11 & $<750$ & $1.0 / 1.0$ & - \\
\hline PD21 & $\mathrm{F}$ & 40 & 8 & - & $1.0 / 1.0$ & - \\
\hline PD22 & M & 67 & 14 & $<500$ & $1.0 / 1.0$ & - \\
\hline PD23 & $\mathrm{F}$ & 55 & 6 & Untreated & $1.0 / 1.0$ & - \\
\hline PD24 & $\mathrm{F}$ & 70 & 18 & $>750$ & $0.8 /-$ & Central venous thrombosis left \\
\hline PD25 & M & 56 & 9 & $<500$ & $1.0 / 1.0$ & - \\
\hline PD26 & M & 37 & 2 & - & $1.0 / 1.0$ & - \\
\hline PD27 & M & 79 & 15 & $<750$ & $0.7 / 0.8$ & - \\
\hline PD28 & M & 59 & 11 & $<500$ & $0.8 / 0.8$ & - \\
\hline PD29 & $\mathrm{F}$ & 72 & 9 & $<375$ & $1.0 / 1.0$ & - \\
\hline PD30 & M & 68 & 17 & $<500$ & $0.8 / 0.8$ & - \\
\hline MSA01 & M & 54 & 11 & $<500$ & $1.0 / 1.0$ & - \\
\hline MSA02 & $\mathrm{F}$ & 63 & 19 & $>750$ & $0.8 / 0.7$ & - \\
\hline MSA03 & $\mathrm{F}$ & 59 & 22 & Untreated & $1.0 / 1.0$ & - \\
\hline MSA04 & $\mathrm{F}$ & 57 & 23 & $<750$ & $0.8 / 0.7$ & - \\
\hline MSA05 & M & 66 & 10 & Untreated & $0.4 / 0.9$ & Ocular hypertension \\
\hline MSA06 & $\mathrm{F}$ & 56 & 23 & $<500$ & $1.0 / 1.0$ & - \\
\hline PSP01 & M & 75 & 4 & $<750$ & $0.8 / 0.8$ & - \\
\hline PSP02 & $\mathrm{F}$ & 68 & - & $<375$ & $0.7 / 0.5$ & Fixational nystagmus \\
\hline PSP03 & M & 68 & 25 & $<750$ & $1.0 / 0.5$ & - \\
\hline PSP04 & M & 61 & 10 & Untreated & $0.8 / 0.7$ & - \\
\hline PSP05 & $\mathrm{F}$ & 69 & 7 & $<750$ & $1.0 / 1.0$ & Artificial lens \\
\hline PSP06 & M & 69 & 19 & $<500$ & $0.8 / 0.8$ & - \\
\hline
\end{tabular}


patients were recruited from the in-patient clinic of the Neurology Department after being informed about the general aims of the investigation. Participation was on a voluntary basis and only occurred after informed consent. Approval for the study was obtained from the local ethical board of the University of Freiburg. Patients with any additional CNS or eye disease were excluded from the study. The clinical diagnosis was made by the ward consultant. A total of 31 patients with idiopathic Parkinson's disease participated (mean age $=58.4$ years; $\mathrm{SD}=9.8$; range: $37-72$ years), 4 of these patients were untreated at the time of study. Possible multiple system atrophy was diagnosed in patients with a Parkinsonian syndrome, who showed little or no response to levo-dopa therapy or cerebellar signs. Probable multiple system atrophy was diagnosed in patients with a Parkinsonian syndrome, who showed little or no levo-dopa response and additional automonic failure, or cerebellar and/or pyramidal signs, or pathological sphincter electromyogram (Quinn, 1994). In our patient sample, two patients were diagnosed as possible multiple system atrophy and 4 patients as probable multiple system atrophy (mean age $=$ 61.7 years; $\mathrm{SD}=8.2$; range: $52-75$ years). A third group of 6 in-patients were diagnosed with progressive supranuclear palsy (mean age $=68.5$ years; $\mathrm{SD}=4.1$; range: $62-75$ ). Diagnostic signs were vertical ocular palsy and/or pseudobulbar palsy, progressive disease course, disease onset after age 40 years, with either axial dystonia, rigidity, bradykinesia, disturbance in stance or gait, or frontal lobe signs (Lees, 1987). The results from these three patient groups were compared to those of a control group $(n=33)$, which was made up of patients with no clinically determined central nervous disorders (mean age $=54.5$ years; $\mathrm{SD}=8.5$; range: $40-72$ years). Care was taken to select control patients so that they were similar to the patient groups with respect to age, gender and socio-economic status. Any further CNS-acting drugs taken by the patients were recorded.

Each patient underwent a detailed ophthalmological examination prior to participation and they wore their full optical correction during testing. Six additional patients were excluded from the study owing to lens opacity or other opthalmological disorders.

\subsection{Stimuli}

The stimulus set-up for electrophysiological and psychophysical testing is presented schematically in Fig. 1. The visual stimuli were created on a Visual Stimulus Generator (VSG 2/3) graphics board (Cambridge Research Systems, Kent, UK) and displayed on a high-resolution color monitor (Eizo; Japan). The voltage determining the luminance of the stimuli was produced with 14-bit digital-to-analogue converters. Correction of the luminance gamma function of the display was calculated using a spectral photometer (Spectra 704; USA) and stored in lookup tables (LUTs). The mean luminance was $40 \mathrm{~cd} / \mathrm{m}^{2}$. The flash stimulus consisted

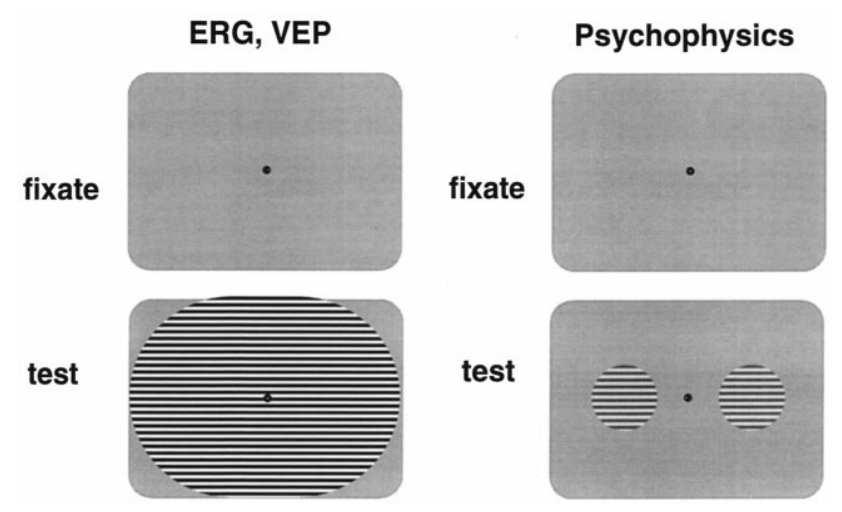

Fig. 1. Schematic illustration of the stimuli used in the experiments. For more details see text.

of a full-field luminance profile that was temporally modulated at $10 \mathrm{~Hz}$. In all other conditions, sinewave gratings were used as stimuli for both electrophysiology and psychophysics. For the psychophysical measurements, circular patches of sinewave grating (diameter $4^{\circ}$ ) with constant orientation (horizontal) and spatial frequency (4 cycles/ deg) but differing in contrast were presented simultaneously for $200 \mathrm{~ms}$, one in each visual field $4^{\circ}$ eccentric to fixation (Fig. 1). Reference contrast levels for psychophysics were $0 \%$ (detection threshold), 10.0, 20.0 and 40.0 and $80.0 \%$ Michelson contrast; for electrophysiology they were 6.25 , $12.5,25,50$, and $100 \%$. Thus, although the contrast values were not identical they spanned a similar range for psychophysics and electrophysiology. The spatial phase of the gratings was temporally modulated at a rate of $6.5 \mathrm{~Hz}$ with a squarewave (electrophysiology) or sinewave (psychophysics) temporal profile.

\subsection{Electrophysiological recordings}

The electroretinogram was recorded in each eye by placing thin wire electrodes (Dawson et al., 1979) into the conjunctival sac near the lower limbus. Reference electrodes were gold cup electrodes filled with electrolytic paste (TECA, Pleasantville, NY) and attached to the outer canthi. This position was selected to minimize the effect of cortical potentials arising along the scalp. Visually evoked potentials were recorded from the scalp at $\mathrm{Pz}$ and referenced to a frontal electrode at FPz (Jasper's 10-20 system). The patient's left ear lobe was grounded. Electrode impedance was measured and was not allowed to exceed $2.5 \mathrm{k} \Omega$. The analogue signals were amplified with a commercially available EEG amplifier system (Toennies, Germany), using a time constant of $0.1 \mathrm{~s}$ and a upper cutoff frequency of $70 \mathrm{~Hz}$. The analogue signals were then passed to analogue-to-digital converters sampling every $2.5 \mathrm{~ms}$. The Fourier spectrum of the resultant signals was computed and the magnitude at the reversal rate was extracted.

The patient fixated a center fixation cross and binocularly viewed, with natural pupils, the patterns while ERGs and 
VEPs were recorded (Fig. 1). The viewing distance was constant at $57 \mathrm{~cm}$. Eye blinks and eye movements were detected via an amplitude threshold $(>100 \mu \mathrm{V})$ and data from these trials were rejected. A minimum of 160 repetitions was conducted per condition.

\subsection{Psychophysical procedure}

All subjects binocularly viewed, with natural pupils, the stimulus display from a distance of $114 \mathrm{~cm}$. The participant was comfortably seated in an examination chair and rested his or her head and arms on appropriately positioned rests. The patients were instructed to direct their gaze to the center of the display, where the fixation cross was displayed during each trial. Each measurement consisted of 150 trials (5 reference contrast levels, 30 trials per level). Each trial was announced by a computer generated sound presented $200 \pm 100 \mathrm{~ms}$ prior to stimulus onset, at which time the fixation cross was also presented. This was followed by simultaneous presentation of reference and test stimuli (Fig. 1). The stimulus duration was $200 \mathrm{~ms}$ and the onset and offset of contrast was abrupt. The participants responded by indicating which stimulus had the higher contrast. In cases where only one stimulus was visible, they were asked to indicate whether the stimulus appeared on the left or right side of the display.

All 4 psychophysical measurements were conducted in a single session. A sufficient number of practice trials was performed to assure that the patient understood the task and became acquainted with the response box (psychophysics). Electrophysiological data were usually collected on the same day.

The methods used to determine psychophysical thresholds have been described earlier (Tebartz Van Elst et al., 1997). Briefly, an adaptive search algorithm, Best-PEST (Lieberman and Pentland, 1982), was used to determine thresholds for each of four reference contrast levels. Each 'staircase' was controlled by the PEST routine, which started at the highest possible test contrast level (approaching $100 \%$ ) and selected one of 80 possible contrast levels.

Reaction times (RT) were also determined using a function that accesses the graphics processor, which timed the duration between the stimulus onset and the response (pressing one of two buttons). We thus could achieve temporal resolution of $10 \mu$ s (i.e. the duration of the buildup of each line on the display). The subjects were given $2 \mathrm{~s}$ to respond. If the subject did not respond within the specified time, the next trial was presented. The lapsed trial was placed back into the trial pool and performed at a later point in the run.

\subsection{Data analyses}

Analyses of variance for repeated measures were performed on the logarithms of detection and discrimination threshold data, as well as on the amplitudes of the ERG and VEP data. Reaction times were also statistically analyzed. The main effects and interactions were determined for the following factors: patient group, spatial frequency, and the reference contrast level (detection threshold, four suprathreshold contrast levels). Correlations between psychophysical, electrophysiological and clinical data were also calculated.

\section{Results}

\subsection{Electroretinograms}

The results for the electroretinographic data are summarized in Fig. 2. The amplitude of the second harmonic response in the electroretinogram is plotted as a function of the stimulus contrast. The leftmost curves show the best-fitting power functions, for patients and controls separately, for the condition of full-field flicker modulation (Flash), the centrally displayed curves show power functions for the low spatial frequency condition $(0.5$ cycles/ degree) and the rightmost curves present these functions for the medium spatial frequency condition (4 cycles/ degree). Over all conditions tested, ERG amplitudes increase with increasing stimulus contrast. The shapes of these 'contrast response' functions varies for the three spatial frequencies tested. Flash ERG amplitudes increase as a compressive function of contrast, whereas pattern ERG amplitude increases as an expansive function of contrast. The derived maximal response levels and exponents are shown in Table 2.

An analysis of variance was performed to assess the effects of experimental group, stimulus contrast and spatial frequency. The eye of acquisition (left, right) had no significant effect on the ERG amplitudes, and is treated as a repeated within-subject variable in the analysis of variance. The main effect of experiment group was highly significant

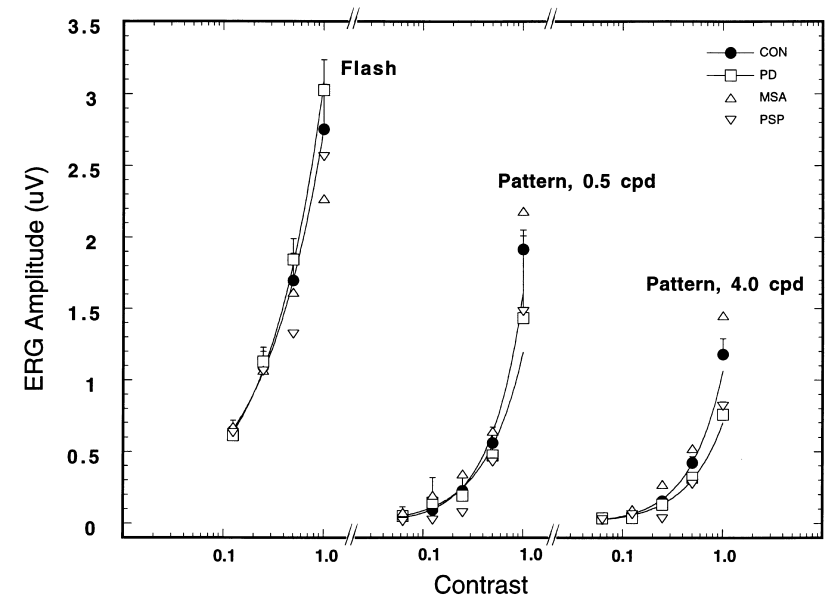

Fig. 2. Amplitude of the second harmonic response in the electroretinogram (ERG) as a function of stimulus contrast (plotted on log scale). The curves show the best fitting power functions for the results from Flash and patterned stimulation (low and high spatial frequency). The different symbols present the results for the four patient groups (see inset). Error bars give $+1 \mathrm{SE}$ 
Table 2

Results of curve fits describing the relationship between ERG amplitude and stimulus contrast. The function fit to the data had the following form: $R=R_{\max } * \mathrm{C}^{\mathrm{a}} ; R_{\max }=$ maximal response (in $\mu \mathrm{V}$ ), $a=$ exponent of power function, $R^{2}=$ coefficient of determination

\begin{tabular}{|c|c|c|c|c|c|c|c|c|c|c|c|c|}
\hline \multirow[t]{2}{*}{ Patient group } & \multicolumn{3}{|l|}{$\mathrm{PD}$} & \multicolumn{3}{|c|}{ MSA } & \multicolumn{3}{|l|}{ PSP } & \multicolumn{3}{|l|}{$\mathrm{CON}$} \\
\hline & $R_{\max }$ & $a$ & $R^{2}$ & $R_{\max }$ & $a$ & $R^{2}$ & $R_{\max }$ & $a$ & $R^{2}$ & $R_{\max }$ & $a$ & $R^{2}$ \\
\hline Flash & 2.75 & 0.744 & 0.998 & 3.54 & 0.806 & 0.999 & 2.90 & 0.819 & 0.992 & 3.2 & 0.702 & 0.999 \\
\hline Pattern $(0.5$ cycles/deg) & 1.27 & 1.14 & 0.979 & 1.37 & 1.256 & 0.997 & 1.04 & 1.02 & 0.975 & 1.7 & 1.265 & 0.984 \\
\hline Pattern (4.0 cycles/deg) & 0.82 & 1.21 & 0.998 & 0.57 & 0.927 & 0.971 & 0.58 & 1.03 & 0.962 & 1.3 & 1.344 & 0.999 \\
\hline
\end{tabular}

$(F(1,71)=8.92 ; P<0.004)$, as were the effects of spatial frequency $(F(2,142)=156.8 ; P<0.0001)$ and stimulus contrast $(F(4,284)=629.7 ; P<0.0001)$. The interaction between the effects of spatial frequency and contrast $(F(8,568)=90.0 ; P<0.0001)$, as well as between experimental group and contrast $(F(4,284)=10.7 ; P<0.0001)$ were highly significant. These interactions substantiate the impression given in Fig. 2 and Table 2 that the contrast response functions differed over spatial frequency and were more shallow for the patients compared to the control subjects. To eliminate any possible effect of visual acuity on the ERG amplitudes, we performed the ANOVA for data from subjects with an acuity of 0.8 or better. This analysis indicated that the main effect of patient group remained significant $(F(1,51)=6.18 ; P<0.017)$. Thus, the effects of Parkinson's disease on ERG amplitudes cannot be explained solely by a loss in visual acuity.

A further analysis of variance was performed to test the effect of the type of Parkinsonian syndrome. Table 2 presents the $R_{\max }$, exponent and correlation values for the three patient groups separately. As above, data collected from either eye were pooled. The main effect of the type of Parkinsonian syndrome was not significant $(F(2,34)=0.34$; n.s. $)$, nor were any of the first- or second-order interactions.

\subsection{Visually evoked potentials}

The results of the visually evoked potential (VEP) analysis are shown in Fig. 3. The amplitude of the response component at the second harmonic frequency is plotted as a function of stimulus contrast. The different symbols present the results of the different patient groups. The functions plot the best fitting hyperbolic ratios, which provide a good description of the relationship between stimulus contrast and neural response (Albrecht and Hamilton, 1982; Sclar et al., 1990). The best fitting values for the three parameters describing this function are given in Table 3. VEP amplitude increases with increasing contrast level. At higher contrast levels the VEP amplitude begins to saturate. There is no obvious difference between the different patient groups. If anything, the patients with Parkinson's disease show slightly larger VEP amplitudes compared to the control patients. Interestingly, the six patients with multiple system atrophy show the largest VEP amplitudes for patterned stimulation.

An analysis of variance was performed to assess the effects of spatial frequency, contrast and patient group on the amplitude of the VEP. This yielded a significant effect of spatial frequency (amplitudes decreasing with increasing spatial frequency; $F(2,6)=3.41 ; P<0.04)$ and contrast (amplitudes increasing with increasing contrast; $F(4,12)=97.8 ; \quad P<0.0001) . \quad$ The interaction term between spatial frequency and contrast was also significant $(F(8,24)=7.0 ; P<0.0001)$, indicating that the contrast response functions differed for the different spatial frequency conditions. Inspection of the results indicated that this effect is mostly related to the difference between the flash and patterned stimulation conditions. The main effect of experimental group (patients vs. controls) was not significant.

\subsection{Contrast thresholds and contrast discrimination}

The results of the psychophysical measurements are shown in Fig. 4, which plots the logarithm of the contrast discrimination threshold as a function of the stimulus contrast. The different symbols present the results for the four patient groups. The fitted functions are hyperbolic

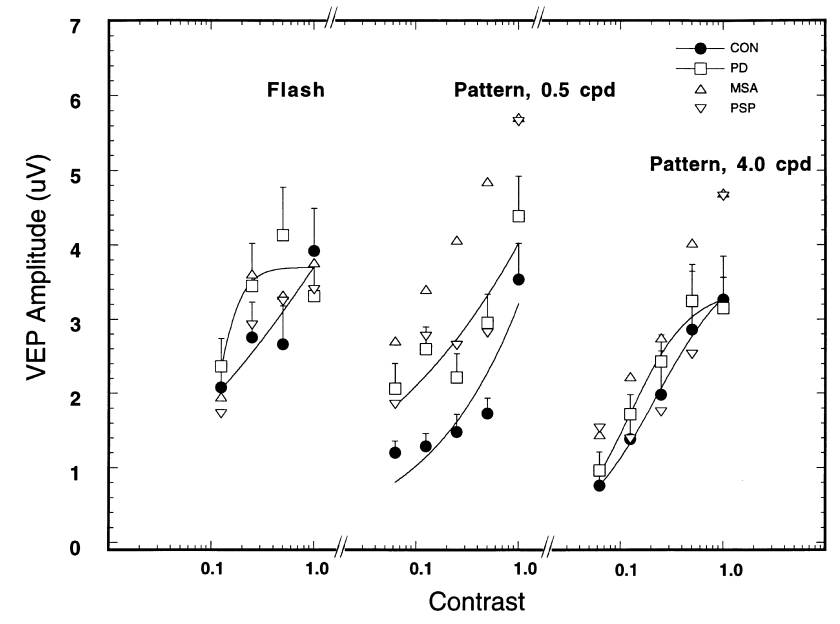

Fig. 3. Amplitude of the visually evoked potential (VEP) measured at the scalp over posterior cortex as a function of the stimulus contrast (log scale). The different curves show the best fitting hyperbolic ratios. Otherwise as in Fig. 2. 
Table 3

Results of curve fits describing the relationship between VEP amplitude and stimulus contrast. The function fit to the data was as follows: $R=R_{\max }\left\{\mathrm{C}^{\mathrm{a}} /\left(\mathrm{C}^{\mathrm{a}}+\mathrm{C}_{50}{ }^{\mathrm{a}}\right)\right\} ; R_{\max }=$ maximal response (in $\left.\mu \mathrm{V}\right), a=$ exponent of hyperbolic ratio, $\mathrm{C}_{50}=$ semi-saturation constant, $R^{2}=$ coefficient of determination

\begin{tabular}{|c|c|c|c|c|c|c|c|c|c|c|c|c|c|c|c|c|}
\hline \multirow[t]{2}{*}{ Patient group } & \multicolumn{4}{|l|}{ PD } & \multicolumn{4}{|l|}{ MSA } & \multicolumn{4}{|l|}{ PSP } & \multicolumn{4}{|l|}{$\mathrm{CON}$} \\
\hline & $R_{\max }$ & $a$ & $\mathrm{C}_{50}$ & $R^{2}$ & $R_{\max }$ & $a$ & $\mathrm{C}_{50}$ & $R^{2}$ & $R_{\max }$ & $a$ & $\mathrm{C}_{50}$ & $R^{2}$ & $R_{\max }$ & $a$ & $\mathrm{C}_{50}$ & $R^{2}$ \\
\hline Flash & 4.9 & 1.2 & 0.7 & 0.999 & 4.91 & 1.21 & 0.813 & 0.999 & 9.35 & 3.38 & 0.839 & 0.99 & 7.7968 & 2.3386 & 0.812 & 0.995 \\
\hline Pattern $(0.5$ cycles $/ \mathrm{deg})$ & 3.28 & 1.18 & 0.999 & 0.99 & 3.87 & 2.35 & 0.9 & 0.987 & 6.08 & 1.64 & 0.99 & 0.99 & 2.5 & 3.23 & 0.71 & 0.993 \\
\hline Pattern (4.0 cycles/deg) & 1.37 & 1.88 & 1.19 & 0.998 & 4.6 & 1.6 & 1.65 & .995 & 1.08 & 3.2 & 0.7 & 0.99 & 1.62 & 2.68 & 0.7 & 0.995 \\
\hline
\end{tabular}

ratios. All groups show contrast discrimination thresholds that increase with increasing stimulus contrast. There is a consistent difference between thresholds measured in the patients with Parkinson's disease compared to the control patients. The elevation in detection thresholds persists in the suprathreshold contrast discriminations. This result confirms an earlier observation from our laboratory (Tebartz Van Elst et al., 1997). Note that the patients with supranuclear palsy have the most difficulty with the psychophysical tasks, whereas patients with multiple system atrophy show results similar to those of the control patients.

An analysis of variance was conducted to examine the main effects of patient group, spatial frequency and contrast level. The effect of group was highly significant $(F(3,58)=4.8 ; \quad P<0.005)$. Post-hoc tests (Bonferroni/ Dunn) indicated that these differences were due to the relatively high contrast thresholds exhibited by the PSP patients (Fig. 4). The effect of spatial frequency was not significant $(F(1,3)=2.2$; n.s. $)$, whereas the effect of contrast was highly significant $(F(4,12)=78.9 ; P<0.0001)$. The interaction between patient group and contrast level was not significant $(F(12,232)=0.7$; n.s. $)$, suggesting that the effect of reference contrast level was similar over the 4 patient groups.

We further examined the possible relationship between

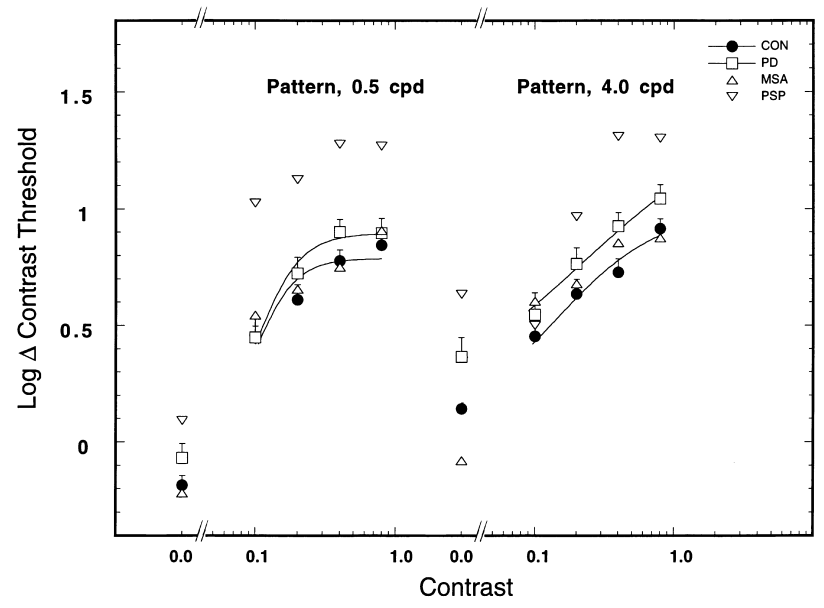

Fig. 4. Log of the contrast discrimination thresholds as a function of the reference contrast on log coordinate. The curves show the best fitting hyperbolic ratios. Symbols and error bars as in Fig. 2. the electrophysiological findings and the psychophysical thresholds. The variables in question are those that significantly differed across the patient groups. Thus we calculated correlation coefficients between the pattern ERG amplitudes at the highest contrast level and the psychophysical thresholds. No significant correlations were found between these variables for the flash and patterned stimulation condition with a low spatial frequency ( 0.5 cycles/degree $)$. There was, however, a significant negative correlation between the ERG amplitudes for 4.0 cycles/degree patterned stimulation at the highest contrast level and the psychophysical thresholds for this spatial frequency $(r=-0.27$; d.f. $=60$; $P<0.03$ ), but not between the VEP amplitude at the highest contrast and thresholds $(r=-0.096$; d.f. $=60$; n.s. $)$. Although the former correlation is only moderate in magnitude, it does suggest that pattern ERG responses and psychophysical thresholds are affected by a common mechanism.

\subsection{Reaction times}

The results of the reaction time measurements are shown in Fig. 5. The logarithm of reaction time (in seconds) is plotted as a function of stimulus contrast for the two conditions of patterned stimulation. As in the other figures, the different symbols present the results of the four patient

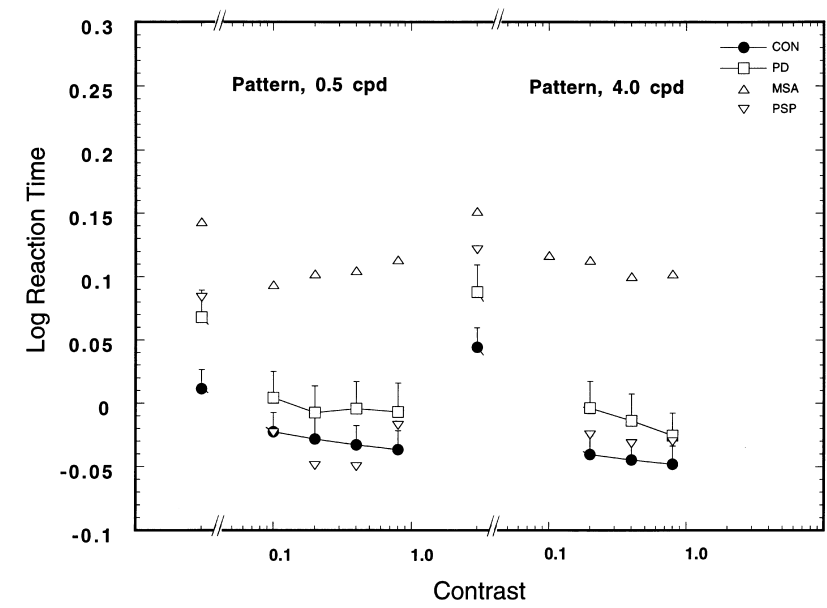

Fig. 5. Log reaction times (RT) as a function of the reference contrast on $\log$ coordinate. Symbols and error bars as in Fig. 3 . 
groups. Reaction times in the detection task with reference contrast equal to zero are shown as the leftmost points for each spatial frequency condition. There is a clear tendency for the patients with multiple system atrophy to exhibit the longest reaction times. Also patients with Parkinson's disease tended to show longer reaction times compared to the control patients. The reaction times are greatest at detection threshold level and decline gradually with increasing suprathreshold contrast.

An analysis of variance revealed a significant main effect of stimulus contrast $(F(4,12)=27.1 ; P<0.0001)$, but no significant effects for spatial frequency $(F(1,3)=0.3$; n.s.) and patient group $(F(3,56)=2.3 ;$ n.s. $)$. Although the differences in log RT look substantial in Fig. 5 the variability across patients is also substantial. As such the trends do not reach significance levels.

\subsection{Correlation with clinical course}

The disease course was evaluated with help of the Webster score (Webster, 1966). The results for each patient are shown in Table 1 . The mean Webster score significantly differed over the patient groups $(F(2,39)=4.3 ; P<0.02)$. The mean Webster score for the patients with Parkinson's disease was $9.6(\mathrm{SD}=5.2)$, for the patients with multiple system atrophy $17.2(\mathrm{SD}=7.4)$ and for the patients with supranuclear palsy $13.75(\mathrm{SD}=9.9)$. The Webster score was used as an index of pathology to correlate with the dependent variables under study here. None of the electrophysiological variables correlated significantly with the Webster score. Significant correlations were, however, evident between the psychophysically determined contrast thresholds and the Webster score (spatial frequency $=0.5$ cylces/degree; $r=0.43, P<0.01$ ). Also the $\log$ of reaction times at threshold significantly correlated with the Webster score (spatial frequency $=0.5$ cylces/degree; $r=0.49$, $P<0.01 ; \quad$ spatial frequency $=4.0 \quad$ cylces $/$ degree; $r=0.48, P<0.01)$. Thus, patients with more severe symptoms tended to perform more poorly in the psychophysical task and required more time to respond.

In our patient sample we had 4 untreated patients, who had not received any L-dopa medication during the investigation. Direct comparisons between untreated and medicated Parkinson's patients are, however, confounded by a number of clinical variables (age, illness duration, etc.). Despite these precautions we compared the most important ERG and psychophysical data between treated and untreated PD patients, but could not find any significant differences. However, due to the small size of the untreated patient group, these observations have to be interpreted with caution.

\section{Discussion}

The present results indicate that the retinal processing of stimulus contrast is impaired in Parkinson's disease. The amplitude of the ERG for low and medium spatial frequencies is significantly reduced in these patients, whereas contrast detection thresholds and contrast discrimination thresholds were significantly elevated. These threshold elevations appear to be a result of an diminished contrast transduction in the retina.

\subsection{Electrophysiological findings}

The amplitudes of the ERGs to patterned stimulation are significantly reduced in Parkinson's disease (Fig. 2). This finding substantiates and extends the earlier results of other groups concerning alterations in the ERG in these patients (Nightingale et al., 1986; Gottlob et al., 1987). The spatial frequency selectivity of the reduction in ERG amplitude (Stanzione et al., 1989; Bodis-Wollner, 1990) points to a specific neural mechanism rather than to a global effect. The more pronounced effect at a spatial frequency of 4 cycles/degree in the present study (Fig. 2) supports this claim.

We also studied the effect of stimulus contrast on the ERG amplitudes to determine the extent to which the contrast response function is altered by the Parkinsonian pathology (Fig. 2). The best fitting power functions yielded accurate approximations of the relationship between ERG amplitude and contrast. For all patients tested these functions were compressive for unpatterned stimulation (flash) and expansive for patterned stimulation (Table 2). The exponents (slopes) of the power functions were consistently lower in the patients with Parkinson's disease and supranuclear palsy compared to the control patients. This effect was much less pronounced in patients with multiple system atrophy, suggesting that the visual system is not affected in this disorder (cf.Tebartz Van Elst et al., 1997). The visually evoked potential recorded over posterior cortex showed a compressive contrast response function (Fig. 3). These functions could be approximated by hyperbolic ratios and the parameters of these functions were presented in Table 3. Overall the VEPs did not significantly differ over the four patient groups studied. This lack of significant difference could be related to the greater variability of amplitudes across patients within each group. It might also reflect the fact that some form of cortical contrast gain control has taken place. The reduced retinal contrast signals could be partially compensated for by cortical mechanisms, which adjust their contrast gain to extract more information. Surely such a compensation can only be partial, since the signal-tonoise ratio will define the limits of perceptual discrimination. In an earlier study (Tebartz Van Elst et al., 1997), we could demonstrate that psychophysical adaptation processes appear to be intact in Parkinson's patients. Such adaptive gain control mechanisms could be useful to ameliorate the effects of impaired retinal signal transmission on vision.

\subsection{Clinical findings and visual pathology}

One of the motivations for the present study was to test 
the usefulness of electrophysiological measures of visual response in the differential diagnosis of Parkinsonian disorders. As we have reported earlier (Tebartz Van Elst et al., 1997), psychophysically determined contrast detection thresholds can differentiate between Parkinson's disease and multiple system atrophy. The results shown in Fig. 4 support this claim. The differences between the patients with Parkinson's disease and multiple system atrophy are, however, less pronounced than those found in our earlier study. We relate this to the finding that the patients with multiple system atrophy in the present study had more pronounced psychomotor impairments, as indexed by significantly higher Webster scores. Since the psychophysical tasks demand attention and vigilance, the more severe disease course in patients with multiple system atrophy could have negatively affected their psychophysical performance. On the other hand, patients with supranuclear palsy exhibited the highest thresholds (Fig. 4) and the highest Webster scores. To our knowledge, this is the first report on contrast sensitivity and visual electrophysiolology on patients with progressive supranuclear palsy. Interpretation of the findings are difficult, since these patients also have impaired oculomotor control, which could have contributed to their relatively poor psychophysical performance. Overall, the findings suggest that electrophysiological measures of visual function could contribute to the differential diagnosis of Parkinson's disease. However, as with other diagnostic tools, these measures should be used together with clinical ratings to differentiate between the different akinetic syndromes.

\subsection{Correlation between electrophysiological and psychophysical measures}

The correlation between the electrophysiological and psychophysical measures was not robust. One critical correlation between ERG amplitude and psychophysical thresholds was significant. This negative correlation indicates that patients with lower ERG amplitudes at maximum contrast exhibit thresholds that are higher than average and vice versa. This trend was significant, however, only for the stimulus with a spatial frequency of 4 cycles/degree. The lack of correlation between the VEP amplitudes and thresholds could be related to the larger noise levels associated with the VEP, but also to the smaller variability - within and across the groups.

\subsection{Relationship to earlier studies}

The spatial-frequency selective reduction in the amplitude of the pattern ERG suggests that the receptive field structure of dopaminergic amacrine, horizontal and interplexiform neurons is altered. Under similar conditions of light adaptation, the ganglion cells of the Parkinsonian retina could display larger central summation areas as a consequence of reduced surround inhibition (Bodis-Wollner, 1990). Ikeda and coworkers put forth a model of the receptoral and postreceptoral processing of visual contrast in the Parkinsonian visual system. They suggest that the interplay between 'on' and 'off' center receptive fields of retinal ganglion cells can be disturbed by the imbalance between dopaminergic, GABAergic and glycinergic receptors in amacrine and interplexiform neurons (Ikeda et al., 1994). Similar concepts have been put forth by other groups to explain the shift in the contrast sensitivity function for detecting sinewave gratings in patients with Parkinson's disease (Bodis-Wollner et al., 1987; Masson et al., 1993) and drug-induced Parkinsonism (Bulens and Meerwaldt, 1989), as well as changes in peripheral (Harris et al., 1992) and perifoveal (Tebartz Van Elst et al., 1997) contrast matching performance in Parkinson's disease.

The alterations in pattern-ERGs can also be accounted for by a deficiency in retinal dopaminergic processing. De novo idiopathic Parkinson's patients exhibit pattern ERGs and VEPs with significantly longer latencies, and this difference is reduced by L-dopa therapy (Peppe et al., 1992; Peppe et al., 1995). Healthy subjects treated with haloperidol, a dopamine antagonist, show pattern ERGs that are more delayed than VEPs (Stanzione et al., 1991). In a 5 year follow-up study of Parkinson's patients (Ikeda et al., 1994), flash and pattern-ERG responses exhibited amplitudes that were more reduced and latencies that were greater at follow-up. The spatial frequency selectivity of the reduction in ERG amplitudes evident in a recent study (Tagliati et al., 1996) and the present one (Fig. 2) support the contention that the retinal dopamine deficiency evident in Parkinson's disease has a specific effect.

The contrast response functions of steady-state VEPamplitudes did not significantly differ between patients with Parkinson's disease and controls (Fig. 3). With transient evoked potentials other groups have found a significant increase in latency (Bodis-Wollner and Yahr, 1978; Gawel, 1981; Mintz et al., 1981). Gottlob et al. (1987) also found significantly increased latencies in transient VEPs in patients with Parkinson's disease, although the amplitudes were within the normal range. It should be noted, however, that an increased latency in the visually evoked cortical response need not imply that the site of this delay is in the visual cortex. To the contrary, the delays found in the ERGs appear to cause the delays found in the cortical VEP.

\subsection{Summary}

We have studied the contrast-amplitude relationships at three levels in the visual system of patients with idiopathic Parkinson's disease and related neurodegenerative disorders. Our findings support and extend those of earlier studies which indicate a reduction in ERG-amplitudes for medium spatial frequencies. A significant decline in the slope of the ERG-amplitude vs. contrast functions in patients with Parkinson's disease, along with the absence of an effect in the visually evoked cortical potential, suggest that the site of the impairment is in the retina. The significant correlation 
between within-subject averaged ERG-amplitudes and psychophysical contrast discrimination thresholds also lends support to this claim.

\section{Acknowledgements}

This research was supported by Hermann and Lilly Schilling Stiftung (to MWG). The authors would like to thank the patients and volunteers for their participation.

\section{References}

Albrecht DG, Hamilton DB. Striate cortex of monkey and cat: contrast response function. J Neurophysiol 1982;48:217-237.

Bodis-Wollner I. Visual deficits related to dopamine deficiency in experimental animals and Parkinson's disease. Trends Neurosci 1990;13:296301.

Bodis-Wollner I, Yahr MD. Measurements of visual evoked potentials in Parkinson's disease. Brain 1978;101:661-671.

Bodis-Wollner I, Marx MS, Mitra S, Bobak P, Mylin L, Jahr M. Visual dysfunction in Parkinson's disease. Loss in spatiotemporal contrast sensitivity. Brain 1987;110:1675-1698.

Bulens C, Meerwaldt JD, Van der Wildt GJ, Keemink CJ. Visual contrast sensitivity in drug-induced Parkinsonism. J Neurol Neurosurg Psychiatry 1989;52:341-345.

Dawson WW, Trick GL, Litzkow CA. Improved electrode for electroretinography. Invest Ophthalmol Vis Sci 1979;18:988-991.

Delwaide PJ, Mesraoua B, De Pasqua V. Les potentiels evoques visuels dans la maladie de Parkinson. Rev Elektroencephal Neurophysiol Clin 1980;10:338-342.

Dowling JE, Ehinger B. Synaptic organisation of amine-containing interplexiform cells of the goldfish and cebus monkey retinas. Science 1975;188:270-273.

Frederick JM, Rayborn ME, Laties AM, Lam DMK, Hollyfield JG. Dopaminergic neurons in the human retina. J Comp Neurol 1982;210:65-79.

Gawel MJ, Das P, Vincent S. Clifford Rose. F. Visual and auditory evoked responses in patients with Parkinson's disease. J Neurol Neurosurg Psychiatry 1981;44:227-232.

Ghilardi, M.F., Marx, M.S., Bodis-Wollner, I. The effect of intraocular 6hydroxydopamine on retinal processing of primates. Ann Neurol 1989;25:357-364.

Gottlob I, Schneider E, Heider W, Skandies W. Alteration of visual evoked potentials and electroretinograms in Parkinson's disease. Electroenceph clin Neurophysiol 1987;66:349-357.

Haggendal J, Malmfors T. Evidence of dopamine containing neurones in the retina of the rabbit. Acta Physiol Scand 1963;64:58-66.

Harnois C. Di Paolo. T. Decreased dopamine in the retinas of patient's with Parkinsons disease. Invest Ophthalmol Vis Sci 1990;31:2473-2475.

Harris JP, Calvert JE, Phillipson OT. Processing of spatial contrast in peripheral vision in Parkinson's disease. Brain 1992;115:1447-1457.

Ikeda H, Head GM, Ellis JK. Electrophysiological signs of retinal dopamine deficiency in recently diagnosed Parkinson's disease. Vis Res 1994;34:2629-2638.

Lees AJ. The Steele-Richardson-Olszewski syndrome (progressive supranuclear palsy). In: Marsden, C.D., Fahn, S. editors. Movement disorders, vol. 2. London: Butterworths, 1987. pp. 272-287.

Lieberman H, Pentland AP. Microcomputer-based estimation of psychophysical thresholds: the best PEST. Behav Res Methods Instrum Comput 1982;14:21-25.
Mariani AP, Kolb H, Nelson R. Dopamine-containing amacrine cells of Rhesus monkey retina parallel rods in spatial distribution. Brain Res 1984;322:1-7.

Marx M, Bodis-Wollner I, Bodak P, Harnois C, Mylin L, Yahr M. Temporal frequency-dependent VEP changes in Parkinson's disease. Vis Res 1986;26:185-193.

Massey SC, Redburn DA. Transmitter circuits in the vertebrate retina. Prog Neurobiol 1987;28:55-96.

Masson G, Mestre D, Blin O. Dopaminergic modulation of visual sensitivity in man. Fund Clin Pharmacol 1993;7:449-463.

Mestre D, Blin O, Serratrice G, Pailhous J. Spatiotemporal contrast sensitivity differs in normal aging and Parkinson's disease. Neurology 1990;40:1710-1714.

Mestre D, Blin O, Serratrice G. Human spatio-temporal contrast sensitivity: dopaminergic induced variations. Eur J Pharmacol 1992;183:10221023.

Mintz M, Tomer R, Radwan H, Myslobodsky MS. Visual evoked potentials in hemiparkinsonism. Electroenceph clin Neurophysiol 1981;52:611616.

Nguyen-Legros J, Botteri C, Le Hoang P, Vigny A, Gay M. Morphology of primate's dopaminergic amacrine cells as revealed by TH-like immunoreactivity on retinal flat mounts. Brain Res. 1988;295:145-153.

Nightingale S, Mitchell KW, Howe JW. Visual evoked cortical potentials and pattern electroretinograms in Parkinson's disease and control subjects. J Neurol Neurosurg Psychiatry 1986;49:1280-1287.

Papadopoulos GC, Parnavelas JG. Distribution and synaptic organisation of dopaminergic axons in the lateral geniculate nucleus of the rat. J Comp Neurol 1990;294:356-361.

Parkinson D. Evidence for a dopaminergic innervation of cat primary visual cortex. Neuroscience 1989;30:171-179.

Peppe A, Stanzione P, Pierelli F, Stefano E, Rizzo PA, Tagliatti M, Morocutti C. Low contrast stimuli enhance PERG sensitivity to the visual dysfunction in Parkinson's disease. Electroenceph clin Neurophysiol 1992;82:453-457.

Peppe A, Stanzione P, Pierelli F, De Angelis D, Pierantozzi M, Bernardi G. Visual alternations in de novo Parkinson's disease. Neurology 1995;45:1144-1148.

Quinn, N. Multiple system atrophy. In: Marsden, C.D., Fahn, S., editors. Movement disorders, vol. 3. Oxford: Butterworth-Heinemann, 1994. pp. 262-281.

Reader TA, Quesney LF. Dopamine in the visual cortex of the cat. Experientia 1986;42:1242-1244.

Sclar G, Maunsell JHR, Lennie P. Coding of image contrast in central visual pathways of the macaque monkey. Vis Res 1990;30:1-10.

Skrandies W, Wässle H. Dopamine and serotin in cat retina: electroretinography and histology. Exp Brain Res 1988;71:231-240.

Stanzione P, Pierelli F, Peppe A, Stefano E, Rizzo PA, Morocutti C, Bernardi G. Pattern visual evoked potentials and electroretinogram abnormalities in Parkinson's disease. Clin Vis Sci 1989;4:115-127.

Stanzione P, Tagliati M, Silverstrini M, Porcu S, Bernardi G. Haloperidol delays pattern electroretinograms more than VEPs in normal humans: a tool in Parkinson's disease pathophysiology. Clin Vis Sci 1991;6:137147.

Tagliati M, Bodis-Wollner I, Yahr MD. The pattern electroretinogram in Parkinson's disease reveals lack of retinal spatial tuning. Electroenceph clin Neurophysiol 1996;100:1-11.

Tebartz Van Elst, L., Greenlee, M.W., Foley, J.M., Lücking, C.H. Contrast detection, discrimination and adaptation in patients with Parkinson's disease and multiple system atrophy. Brain 1997;120:2219-2228.

Webster DD. Clinical analysis of the disability in Parkinson's disease. Mod Treatment 1966;5:257-282. 